'Departamento de Biología, Universidad de Tarapacá, Arica, Chile. ${ }^{2}$ Departamento de Salud Pública, Pontificia Universidad Católica de Chile, Santiago, Chile. ${ }^{3}$ División de Promoción y Políticas Saludables, Ministerio de Salud, Santiago, Chile.

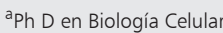
y Genética (Universidad Autónoma de Madrid).

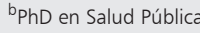
(Universidad de Chile). IIngeniero Civil Sanitario (Universidad de Chile).

dMédico, Magíster en Salud Pública (Universidad de Chile).

Los autores declaran que no existe conflicto de intereses en la ejecución de este trabajo. Las instituciones involucradas no tuvieron influencia en el diseño del estudio, recolección,

análisis e interpretación de los datos y la aprobación de este manuscrito. El estudio fue financiado parcialmente por el Ministerio de Salud de Chile y por el Proyecto $N^{\circ} 4705$ de la Universidad de Tarapacá

Recibido el 25 de enero de 2010, aceptado el 19 de noviembre de 2010

Correspondencia a: Dra. Catterina Ferreccio Fax 6331840 E-mail: cferrec@med.puc.cl

\section{Análisis de las variables del espermiograma en jóvenes sanos en Arica-Chile}

\author{
OMAR ESPINOZA-NAVARRO ${ }^{1, \mathrm{a}}$, SANDRA CORTÉS A. 2,b, \\ JULIO MONREAL ${ }^{3, \mathrm{c}}$, CATTERINA FERRECCIO $^{2, \mathrm{~d}}$
}

\section{Spermograms of healthy young subjects living in Arica, Chile}

Background: Semen analysis is one of the parameters used to predict male fertility. Semen can be altered by environmental pollutants; therefore it could be used as a biological marker of exposure in contaminated areas. Aim: To analyze the spermogram values in a sample of healthy young males, residing in Arica, Chile. Material and methods: One hundred and two healthy university students volunteers aged 18 to 30 years answered a questionnaire about fertility, habits and andrologic diseases and provided a semen sample. Within three hours after ejaculation, semen volume, $p H$, sperm concentration, motility and morphology were analyzed. Results: Six percent of volunteers had offspring, $1 \%$ declared to be infertile, 32\% smoked and $78 \%$ consumed alcohol. Semen $\mathrm{pH}$ was $7.6 \pm 0.5$, volume, $2.9 \pm 1.6 \mathrm{ml}$, sperm concentration, $62.8 \pm 62.3 \times 10^{6} / \mathrm{ml}$, normal morphology, $15.0 \pm 7.9 \%$, overall motility, $42.2 \pm 23.2$ $\%$ and grade A motility, $19.2 \pm 18.6 \%$. The percentage of subjects that had normal semen values was $82 \%$ for total sperm count, $76 \%$ for sperm concentration, $72 \%$ for volume, $64 \%$ for vitality, $63 \%$ for $\mathrm{pH}$, 57\% for morphology, 38\% for overall motility and $26 \%$ for grade A motility. Conclusions: This sample of healthy young males had a normal sperm count in comparison with international reports. However it is necessary to characterize the spermogram in uncontaminated areas of Chile to ensure that our results are within the expected values for the country.

(Rev Med Chile 2010; 138: 1510-1516).

Key words: Fertility; Sperm count; Spermatozoa.
E 1 análisis de la calidad del semen es considerado un elemento básico en la evaluacion de la fertilidad masculina ${ }^{1-3}$. Los parámetros mínimos para evaluar calidad del semen incluyen volumen seminal, $\mathrm{pH}$, concentración, motilidad, viabilidad y morfología espermática, siendo la concentración, motilidad y morfología espermática, los parámetros más importantes ${ }^{4-7}$. Las características del semen varían según la edad, patologías asociadas, exposición a diversos contaminantes ambientales químicos, temperatura corporal y ambiental, sector geográfico, luminosidad y consumo de alcohol y tabaco ${ }^{8-11}$.
La Organización Mundial de la Salud (OMS) ha desarrollado un manual de laboratorio para el examen de semen ${ }^{12}$, recomendando que cada laboratorio de reproducción determine sus propios valores de referencia para los parámetros seminales. Los intentos de comparación internacional fallan a menudo porque hay importantes diferencias en los métodos de reclutamiento de los sujetos en los diferentes países, considerando algunos autores que estos criterios no se aplican a su realidad ${ }^{7,13}$.

Recientemente, el estudio de los parámetros del espermiograma se considera un importante marcador biológico para evaluar la exposición 
ambiental y ocupacional a diferentes agentes químicos, destacándose como un metódo de medición no invasivo para medir productos tales como cadmio, mercurio, plomo, boro, contaminantes clorados y bromados y pesticidas, observándose asociaciones entre estos agentes químicos y parámetros seminales alterados ${ }^{14-18}$.

En Chile, no hay estudios relacionados con parámetros seminales en población joven sana que permitan establecer valores de referencia de estos parámetros en población chilena. Se decidió establecer las características seminales de una población de hombres jóvenes aparentemente sanos residentes en la ciudad de Arica, para posteriormente compararlos con otras poblaciones internacionales expuestas a diferentes contaminantes ambientales. Este es el primer estudio de este tipo en el país y uno de los pocos en población latina ${ }^{8}$. Este estudio forma parte de una investigación más amplia sobre contaminación ambiental y salud realizada en la ciudad de Arica y financiada por el Ministerio de Salud de Chile. La ciudad de Arica se caracteriza por la ocurrencia simultánea de exposicion poblacional a boro en el agua potable y a otros agentes químicos en el suelo.

Este estudio fue aprobado por los Comités de Ética del Servicio de Salud Zona Norte, Coquimbo y de la Universidad de Tarapacá.

\section{Material y Método}

\section{Universo y sujetos de estudio}

El universo en estudio corresponde a 400 varones de entre 18 a 30 años de edad, inscritos en dos carreras de la Universidad de Tarapacá, Arica, Chile $\left(13^{\circ} 28^{\prime}\right.$ latitud sur y $70^{\circ} 20^{`}$ longitud oeste). El tamaño muestral a conveniencia fue de 100 sujetos (25\% del universo), más $10 \%$ para compensar pérdidas en el muestreo. Se inició con un pilotaje de los métodos en una muestra de 10 sujetos, los que no fueron considerados en este análisis. Se invitó a todos los estudiantes a participar en el estudio, incorporándose secuencialmente a los voluntarios, hasta completar el tamaño muestral deseado. A los sujetos se les explicó las características del estudio y se les solicitó la firma de consentimiento informado. A los aceptantes se les dió instrucciones para la toma de las muestras y se les citó al Laboratorio de Biología de la Reproducción de la Universidad de Tarapacá, para realizar el examen seminal y responder un cuestionario epidemiológico y clínico.

Se excluyeron del estudio los sujetos con patologías andrológicas (varicocele ocriptorquidia) previas que pudieran afectar el espermiograma.

\section{Toma y análisis de muestras de semen}

Las muestras de semen fueron obtenidas por masturbación y eyaculación y recibidas en un frasco de polietileno de $50 \mathrm{ml}$, de boca ancha, cerrado y estéril. La única toma se realizó en una sala especialmente habilitada en condiciones de privacidad. Las muestras fueron tomadas después de 4 días de abstinencia sexual.

Inmediatamente de recibida la muestra, se llevó al laboratorio donde se procedió al examen macroscópico y microscópico según método de OMS (1999) y de la European Society of Human Reproduction and Embryology (ESHRE, 2002) ${ }^{12-19}$.

Las muestras fueron colocadas en estufa a 37 ${ }^{\circ} \mathrm{C}$, por 20 minutos para su licuefacción y luego, a los 60 minutos, se analizó volumen, $\mathrm{pH}$, color, olor, consistencia, aspecto, licuefacción, recuento espermático, concentración espermática total, motilidad espermática, vitalidad espermática y morfología.

Para el recuento espermático se utilizó una muestra de $20 \mu \mathrm{L}$ (1:10) en una solución de formaldehído y eosina amarilla y luego se procedió al recuento en cámara hemocitométrica de Neubauer. El recuento espermático total fue calculado como el producto del volumen seminal y la concentración espermática. La motilidad espermática se determinó en una muestra directa de $10 \mu \mathrm{L}$ y se midieron los porcentajes de espermatozoides rápidos (A), lentos (B), no progresivos (C) e inmóviles (D). La vitalidad se evaluó usando el método de tinción de la eosina-nigrosina, determinando el porcentaje de espermatozoides vivos no teñidos. Para la morfología espermática las muestras fueron fijadas y teñidas con el método de Papanicolaou y analizadas con criterio estricto (ESHRE, 2002) ${ }^{19}$. Los análisis de motilidad, vitalidad y morfología espermática se evaluaron en 200 espermatozoides. Las muestras de semen fueron descartadas después de realizados los análisis.

\section{Cuestionario epidemiológico y clínico}

Se aplicó un cuestionario que exploró antecedentes de fertilidad, hábito tabáquico, ingesta de agua y otros líquidos, fármacos, consumo de 
alcohol y drogas; además de enfermedades andrológicas, tales como hernia inguinal, varicocele, epididimitis, gonorrea, infección por clamidias, testículo no descendido o cirugía por torsión testicular. También se recogió información de estados febriles durante los últimos tres meses.

\section{Análisis de los datos}

Las características demográficas de los participantes se expresaron como proporciones, en relación al total de sujetos.

En cuanto a los parámetros del espermiograma, éstos se analizaron como variables continuas de manera separada, mediante gráficos de dispersión de puntos y su distribución por deciles. Se muestran los estadígrados más relevantes, esto es, media y desviación estándar, mediana y rango. A su vez, se determinó el porcentaje de sujetos que superó el nivel de normalidad para cada parámetro, tomando como referencia los puntos de corte establecidos por la OMS para $\mathrm{pH}$, volumen, concentración espermática, recuento total, motilidad grado $\mathrm{A}, \mathrm{y}$ motilidad total $(\mathrm{A}+\mathrm{B}) \mathrm{y}$ vitalidad. Para morfología el punto de corte correspondió al criterio estricto de Tygerberg. No se identificaron diferencias entre grupos.

Los resultados de este estudio se compararon con resultados de otros estudios extranjeros realizados en población general y se presentan medias, desviación estándar y rangos.
Los datos de cada participante se ingresaron y analizaron utilizando el software estadístico SPSS para Windows, versión 14 (SPSS Inc, Chicago, III, 2006) ${ }^{20}$.

\section{Resultados}

Aceptaron participar en el estudio 109 sujetos (27,3\% del universo), siete voluntarios fueron excluidos por presentar historial andrológico alterado, de ellos dos presentaron criptorquidia y cinco varicocele. El análisis se realizó con 102 sujetos elegibles con muestra suficiente y un historial andrológico normal. Sólo 6 participantes reportaron haber tenido hijos $(5,9 \%)$, dos presentaron problemas reproductivos propios o de su pareja y cuatro reportaron problemas de fertilidad de sus padres. 32,4\% de los participantes reportó ser fumador activo y $78,4 \%$ consumir alcohol (Tabla 1).

En la Tabla 2 se presentan los valores de cada parámetro del espermiograma y el cumplimiento de los criterios de normalidad para dichos parámetros. Los valores medios de $\mathrm{pH}$, volumen, concentración y morfología espermática (criterio estricto de Tygerberg), están dentro de los rangos de normalidad. En tanto los patrones de motilidad grado A y motilidad total $(A+B)$, son más bajos que los indicados por OMS.

Tabla 1. Caracterización de la muestra de varones entre 18 a 30 años, Arica-Chile, 2007

\begin{tabular}{|c|c|c|}
\hline Característica & $\mathbf{n}$ & Proporción \% \\
\hline Tamaño de la muestra & 109 & 100 \\
\hline Número de sujetos excluidos & 7 & 6,4 \\
\hline $\begin{array}{l}\text { Criterios de exclusión } \\
\text { Criptorquidia } \\
\text { Varicocele }\end{array}$ & $\begin{array}{l}2 \\
5\end{array}$ & $\begin{array}{l}1,8 \\
4,6\end{array}$ \\
\hline Sujetos elegibles & 102 & 94,5 \\
\hline $\begin{array}{l}\text { Antecedentes reproductivos } \\
\text { Voluntarios que han tenido hijos } \\
\text { Problemas reproductivos en participantes o pareja } \\
\text { Problemas reproductivos en los padres }\end{array}$ & $\begin{array}{l}6 \\
2 \\
4\end{array}$ & $\begin{array}{l}5,9 \\
2,0 \\
3,9\end{array}$ \\
\hline Hábitos & & \\
\hline Fumador activo (prevalencia actual) & 33 & 32,4 \\
\hline Consumo de alcohol & 80 & 78,4 \\
\hline
\end{tabular}


Espermiograma en jóvenes sanos - O. Espinoza-Navarro et al

Tabla 2. Características del análisis de semen en 102 varones. 18-30 años. Arica-Chile, 2007

\begin{tabular}{|lccccc|}
\hline Criterio & Valores OMS & $\begin{array}{c}\text { \% cumple } \\
\text { criterios }\end{array}$ & Media \pm DE & Mediana & Rango \\
\hline $\mathrm{pH}$ & $\geq 7,2$ & 62,7 & $7,6 \pm 0,5$ & 8 & $7-8$ \\
Volumen & $\geq 2$ & 72,5 & $2,9 \pm 1,6$ & 2,6 & $0,3-8,5$ \\
\hline Concentración (x 10\%/ml) & $\geq 20$ & 75,5 & $62,8 \pm 62,3$ & 43,5 & $0,45-380$ \\
\hline Recuento total (x 106) & $\geq 40$ & 82,4 & $170,5 \pm 181,4$ & 112,5 & $1,71-1.140$ \\
\hline Motilidad total A+B (\%) & $\geq 50$ & 38,2 & $42,2 \pm 23,2$ & 42,5 & $0-90$ \\
Motilidad grado A (\%) $^{2}$ & $\geq 25$ & 26,5 & $19,2 \pm 18,6$ & 14,6 & $0-85$ \\
\hline Vitalidad (\%) & $\geq 75$ & 63,3 & $77,9 \pm 19,2$ & 85 & $0-100$ \\
\hline Morfología $^{3}$ & $\geq 14^{*}$ & 56,9 & $15,0 \pm 7,9$ & 15 & $0-43,1$ \\
\hline
\end{tabular}

*Referencia corresponde a criterio estricto de Tygerberg. ${ }^{1-2-3}$ Criterios importantes del espermiograma en la determinación de infertilidad. ${ }^{1}$ Concentración; ${ }^{2}$ motilidad; ${ }^{3}$ morfología.

Los criterios que se cumplen en la mayoría de los sujetos fue el recuento total $(82,4 \%)$, la concentración $(75,5 \%)$, volumen $(72,5 \%)$, la vitalidad $(63,7 \%), \mathrm{pH}(62,7 \%)$ y morfología $(56,9 \%)$. El criterio con menor cumplimiento fue el de motilidad, donde motilidad grado A presenta valores de $26,5 \%$ y el de motilidad total $(A+B) 38,2 \%$ (Tabla 2).

Estos resultados se compararon con los obtenidos en otros estudios de poblaciones de adultos jóvenes sanos de varios países entre los años 2000 al 2008 (Tabla 3).

\section{Discusión}

Varias características del espermiograma promedio de la población joven sana de Arica superaban los estándares recomendados por la OMS (volumen, concentración, recuento total, vitalidad). Sólo la motilidad fue menor al criterio OMS, alcanzando $84 \%$ de cumplimiento. Este parámetro también ha sido encontrado por debajo lo considerado normal por OMS en otras poblaciones $^{5-8,10-13,21-27}$.

Entre los factores que alteran el espermiograma se describen patologías andrológicas asociadas, hábitos y estilos de vida. En nuestro estudio las prevalencias de criptorquidia y varicocele fueron de $1,8 \%$ y $4,6 \%$ respectivamente, valores más bajos que lo reportado en estudios internacionales $^{23,25,28,29}$. Las prevalencias de consumo de alcohol y tabaco son altas $(78,4 \%$ y $32,4 \%$ respectivamente) sin embargo, son similares a las prevalencias del resto de Chile ${ }^{8,9,31,32}$.

Algunos autores estiman que la motilidad y la concentración son mejores predictores de fertilidad que la morfología espermática ${ }^{33}$, mientras que otros dan mayor importancia a la morfología o a la cinética de desplazamiento del espermatozoide ${ }^{34,35}$. Ho et al (2007) demostró que existe asociación entre la morfología según criterio estricto y la fertilidad, siendo mucho menos importantes el volumen y la concentración espermática ${ }^{25}$. Parte de estas discrepancias se pueden deber a que la medición de estos parámetros es subjetiva, dificultando la comparabilidad entre estudios; la OMS (1999) y el Centro Hospitalario de Tygerberg han propuesto métodos y criterios estandarizados que son los que se aplicaron en este estudio así como en aquellos con los que comparamos nuestros resultados ${ }^{12,19,21,34}$. Así, los espermiogramas de los jóvenes de Arica fueron similares en cuanto al volumen, concentración y recuento total a otros estudios comparables realizados en Latinoaméri$\mathrm{ca}^{8,28}$. Actualmente, no es posible comparar con otros estudios nacionales porque en Chile sólo se han reportado estudios clínicos en pacientes infértiles y estudios de casos de donantes de semen ${ }^{36,37}$.

Si bien en los estudios clínicos de fertilidad hay consenso en que éstos se deben iniciar con un examen del espermiograma, este no es suficiente para dar cuenta del éxito reproductivo a nivel de pareja, por lo cual siempre se complementa con 


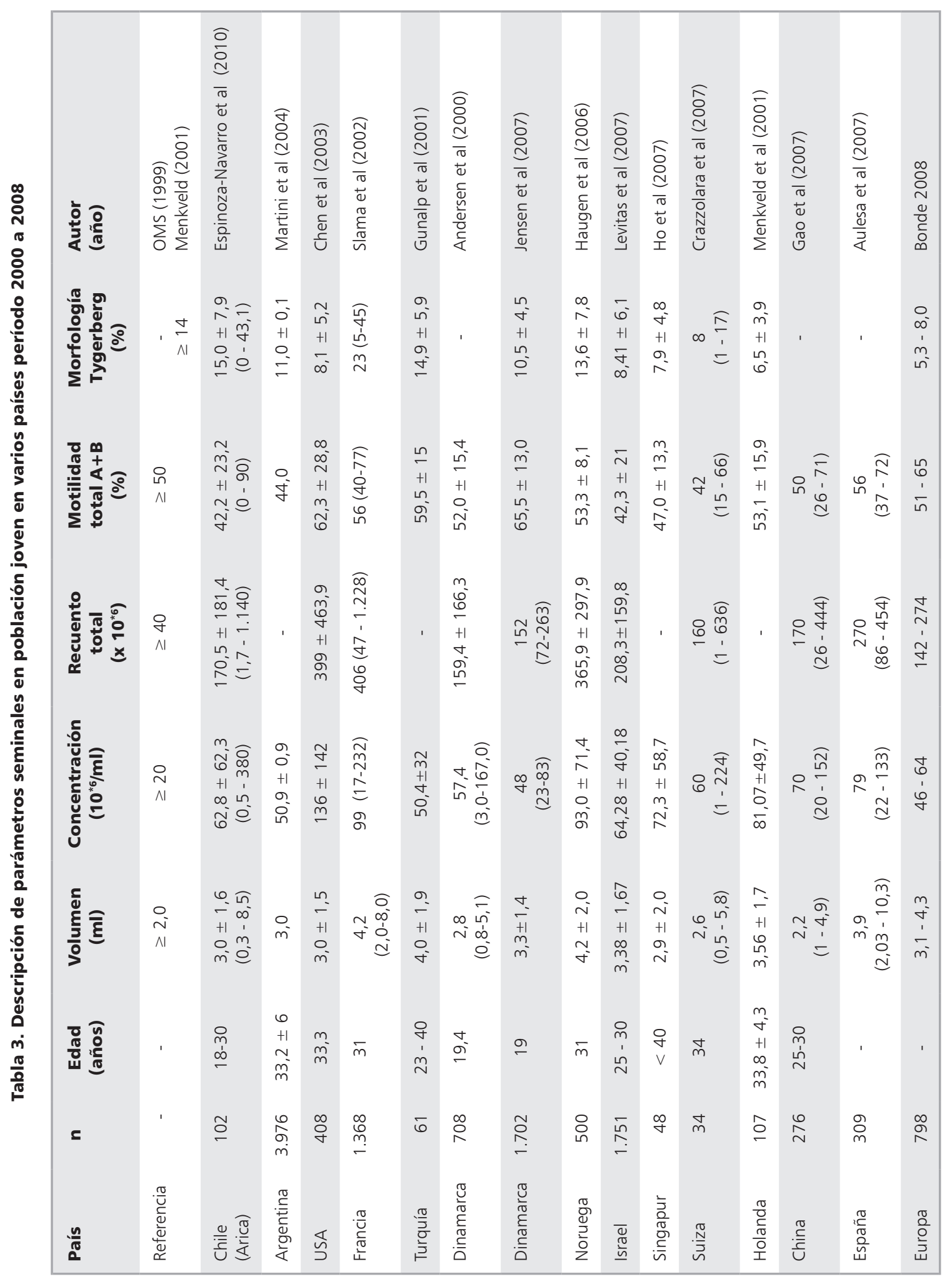


otras pruebas y exámenes clínicos ${ }^{6,12,21}$. Por ello, un examen aislado de espermiograma no puede ser usado para un diagnóstico clínico. Sin embargo, un espermiograma único, tomado en condiciones estándarizadas, da cuenta de la situación media de un grupo en un momento determinado. Así, en promedio, el grupo estudiado en Arica muestra un espermiograma normal, sugiriendo que no habría una alteración significativa de éste biomarcador a nivel poblacional. Como se indicara previamente, esta normalidad es asignada por la comparación de nuestros resultados con aquellos de otros estudios realizados en condiciones similares en poblaciones jóvenes de diversos continentes. Es necesario caracterizar el espermiograma en la población chilena joven residente en zonas libres de contaminación que son el mejor patrón poblacional de comparación de los jóvenes de Arica. Dicho análisis permitirá asegurar que los varones de la ciudad de Arica no presentan un deterioro en su capacidad reproductiva respecto a zonas sin contaminación ambiental. En la encuesta original no se consultó sobre tiempo de residencia en la ciudad, una encuesta complementaria aplicada a todos los alumnos de los cursos participantes, mostró que el 70\% nació y reside en Arica, y que el 30\% nació en otras comunas y reside en Arica por dos o más años. De modo que los participantes presentan entre 2 y 30 años de residencia en la ciudad.

Agradecimientos: Al Dr. Eduardo BustosObregón, Facultad de Medicina, Universidad de Chile, quién nos proporcionó la sección del cuestionario utilizada para evaluar antecedentes clínicos andrológico de los participantes.

\section{Referencias}

1. Tomlinson M, Kessopoulou E, Barratt C. The diagnostic and prognostic value of tradicional semen parameters. J Androl 1999; 20: 588-93.

2. Van der Merve F, Kruger T, Oehninger S, Lombard C. The use of semen parameters to identify the subfertile male in the general population. Gynecol Obstet Invest 2005; 59: 86-91.

3. Lewis S. Is sperm evaluation useful in predicting human fertility? Reproduction 2007; 134: 31-40.

4. Bonde JP, Ernst E, Jensen TK, Hjollund NH, Kolstad H, Henriksen TB, et al. Relation between semen quality and fertility: a population-based study of 430 first-pregnancy planners. Lancet 1998; 352 (9135): 1172-7.
5. Andersen AG, Jensen TK, Carlsen E, Jorgensen N, Andersson AM, Krarup T, et al. High frequency of suboptimal semen quality in an unselected population of young men. Hum Reprod 2000; 15: 366-72.

6. Gunalp S, Onculoglu C, Gurgan T, Kruger TF, Lombard CJ. A study of semen parameters with emphasis on sperm morphology in a fertile population: an attempt to develop clinical thresholds. Hum Reprod 2001; 16: 110-4.

7. Haugen TB, Egeland T, Magnus O. Semen parameters in Norwegian fertile men. J Androl 2006; 27: 66-71.

8. Martini A, Molina R, Estofán D, Senestrari D, Fiol de Cuneo M, Ruiz D. Effects of alcohol and cigarette consumption on human seminal quality. Fertil Steril 2004; 82: 374-7.

9. Ramlau-Hansen C, Thulstrup A, Aggerholm A, Jensen $\mathrm{M}$, Toft $\mathrm{G}$, Bonde JP. Is smoking a risk factor for decreased semen quality? A cross-sectional analysis. Human Reprod 2007; 22: 188-96.

10. Gao J, Gao ES, Yang Q, Walker M, Wu JQ, Zhou WJ, et al. Semen quality in a residential, geographic and age representative sample of healthy Chinese men. Hum Reprod 2007; 22: 477-84.

11. Levitas E, Lunenfeld E, Weisz N, Friger M, Potashnik G. Relationship between age and semen parameters in men with normal sperm concentration: analysis of 6022 semen samples. Andrología 2007; 39: 45-50.

12. World Health Organization (WHO). Laboratory Manual for the examination of human semen and sperm-cervical mucus interaction. (1999) $4^{\text {th }}$ ed. Cambridge, United Kingdom: Cambridge University Press.

13. Crazzolara S, Wunder D, Nageli E, Bodmer C, Graf S, Birkhauser MH. Semen parameters in a fertile Swiss population. Swiss Med Wkly 2007; 137(11-12): 166-72.

14. Giudice LC. Infertility and the environment: the medical context. Semin Reprod Med 2006; 24: 129-33.

15. Comhaire FH, Mahmoud AM, Schoonjans F. Sperm quality, birth rates and the environment in Flanders (Belgium). Reprod Toxicol 2007; 23: 133-7.

16. Xing $\mathrm{X}, \mathrm{Wu}$ G, Wei F, Liu P, Wei H, Wang C, et al. Biomarkers of environmental and workplace boron exposure. J Occup Environ Hygiene 2008; 5: 141-7.

17. Esteban M, Castaño A. Non-invasive matrices in human biomonitoring: A review. Environ Int 2009; 35: 438-49.

18. Hauser R. The environment and male fertility: recent research on emerging chemicals and semen quality. Semin Reprod Med 2006; 24: 156-67.

19. European Society of Human Reproduction and Embryology (ESHRE). Monographs. Manual on Basic Semen Analysis (2002). Ed. U. Kvist and L. Bjorndahl. Oxford University Press. 
20. SPSS Inc. (2006). Advanced statistics for Windows, version 14.0. Chicago, EE.UU.

21. Menkveld R, Wong W, Lombard C, Wetzels A, Thomas $\mathrm{C}$, Merkus $\mathrm{H}$, et al. Semen parameters including WHO and strict criteria morphology in a fertile and subfertile population: an effort towards standardization of in-vivo thresholds. Human Reprod 2001; 16: 1165-71.

22. Chen Z, Toth T, Godfrey-Bailey L, Mercedat N, Schiff I, Hauser R. Seasonal variation and age-related changes in human semen parameters. J Androl 2003; 24: 226-31.

23. Slama R, Eustache F, Ducot B, Jensen T, Jorgensen N, Horte A, et al. Time to pregnancy and semen parameters: a cross-sectional study among fertile couples from four European cities. Human Reprod 2002; 17: 503-15.

24. Jensen T, Jorgensen N, Asklund C, Carlsen E, Holm M, Skakkebaek N. Fertility treatment and reproductive health of male offspring: A study of 1,925 young men from the general population. Am J Epidemiol 2007; 165: 583-90.

25. Ho LM, Lim AS, Lim TH, Hum SC, Yu SL, Kruger TF. Correlation between semen parameters and the Hamster Egg Penetration Test (HEPT) among fertile and subfertile men in Singapore. J Androl 2007; 28: 158-63.

26. Aulesa C, Lasheras J, Gris J, Herrero J, Márquez C, Iglesias A. Estudio de las variaciones estacionales de los parámetros analíticos del seminograma. Rev Int Androl 2007; 5: 337-42.

27. Bonde JP, Toft G, Rylander L, Rignell-Hydbom A, Giwercman A, Spano M, et al. Fertility and markers of male reproductive function in Inuit and European populations spanning large contrasts in blood levels of persistent organochlorines. Environ Health Perspect 2008; 116: 269-77.

28. Smith GR, Kaune GH, Parodi ChD, Madariaga AM, Morales DI, Ríos SR, et al. Aumento del daño en el ADN y estrés oxidativo en espermatozoides de pacientes con oligozoospermia idiopática y antecedentes de criptorquidismo. Rev Med Chile 2007; 135: 279-86.

29. Hadziselimovic F, Hocht B, Herzog B, Buser MW. Infertility in cryptorchidism is linked to the stage of germ cell development at orchidopexy. Horm Res 2007; 68: 46-52.

30. Homan GF, Davies M, Norman R. The impact of lifestyle factors on reproductive performance in the general population and those undergoing infertility treatment: a review. Hum Reprod Update 2007; 13: 209-23.

31. Ministerio de Salud de Chile (Minsal) 2006. Segunda Encuesta Nacional de Calidad de vida y Salud. Disponible en: http://epi.minsal.cl/epi/html/invest/ENS/InformeFinalENS.pdf. Revisada el 30 de octubre del 2008.

32. Consejo Nacional para el Control de Estupefacientes (CONACE), 2003. Informe sobre uso, abuso y dependencia al alcohol. V Estudio Nacional de Drogas en Población General de Chile. 2002. http://www.conace. gov.cl/inicio/index.php.

33. Nallella K, Sharma R, Aziz N, Agarwal A. Significance of sperm characteristics in the evaluation of male infertility. Fertil Steril 2006; 85: 629-34.

34. Eustache F, Auger J. Inter-individual variability in the morphological assessment of human sperm: effect of the level of experience and the use of standard methods. Hum Reprod 2003; 18: 1018-22.

35. Munuce M, Cardona-Maya W, Berta C. ¿Existe asociación entre la morfología normal del espermatozoide y su cinética de desplazamiento? Actas Urológicas Españolas 2006; 591-7.

36. Devoto CE, Madariaga AM, Lioi CX. Factores causales de infertilidad masculina: Contribución del factor endocrino. Rev Med Chile 2000; 128 (2): 184-92.

37. Sánchez GR, Peña SP, Miska W, Henkel R. Determinación de la variabilidad en la condensación de la cromatina espermática y alfa-glucosidasa seminal con relación al espermiograma. Rev Med Chile 2000; 128: 483-9. 